\title{
23. CHEMICAL STRATIGRAPHY OF LEG 45 BASALTS: ELECTRON PROBE ANALYSES OF GLASSES
}

\author{
William G. Melson, Department of Mineral Sciences, Smithsonian Institution, Washington, D.C.
}

\section{INTRODUCTION}

One of the first steps in interpreting oceanic basement cores of pillow lavas is establishing the chemical stratigraphy of the sequences. Sequences with identical chemical composition and without lithologic breaks, such as sequences of sediments, metasediments, or of iron-manganese oxide-encrusted zones, are in all probability single flows. If analogies with land basaltic eruptions are valid, such units probably were deposited over a short time interval, on the order of hours to a few days, and certainly less than several years.

During Leg 45 , the chemical stratigraphy was tentatively established by obtaining X-ray fluorescence analysis (XRF: Bougault, this volume) of at least one rock from each core and from above and below each obvious lithologic break. By this method, eleven chemical types were discovered from the three holes that were cored-two from Hole 395, ten from Hole 395A (two of which correlate with Hole 395), and one from Hole 396. Thickness of these units ranges from 7 to 207 meters.

This paper examines the chemical stratigraphy of Leg 45 by using electron microprobe analyses (EMX) of glassy rinds. Petrogenetic relationships and comparisons with other MORB (Mid-Ocean Ridge Basalt) are not included in the paper. The analytical methods and the usefulness of this approach have been described in various papers and recently reviewed (Byerly et al., 1977). The chemical stratigraphy established on the basis of these analyses further substantiates the chemical stratigraphy established on board ship; in addition, three new chemical types are established for Hole $395 \mathrm{~A}$, and two shipboard types, $\mathrm{P}_{1}$ and $\mathrm{P}_{2}$, Hole 395A (Bougault, this volume) are shown to have identical glass compositions in the analyzed samples. In all, sixty chemical analyses are given for nine elements each (Tables 1, 2, 3, and 4).

This paper concerns itself with primary magmatic units. Breccias are not described or interpreted here.

\section{ANALYTICAL METHODS AND PRECISION}

A major area of basement core study involves correlations of chemical basalt units with magnetic polarity and lithologic changes. Compositional differences between adjacent chemical units can be small, and recognition of their contact requires high-precision chemical analyses. Electron probe analyses of glass are more and more being used as a tool for such discrimi- nation. An assessment of their precision or level of discrimination is thus useful, as well as the electron probe operation conditions.

Glass analyses have a precision closely comparable to the shipboard XRF analyses, and have the advantage of not reflecting compositional changes produced by alteration effects or inhomogeneous distribution of phenocrysts.

Glasses were analysed using a 20-micron electron beam to avoid alkali volatilization. Beam current was 30 microamps. Lower currents were found to give lower precision, higher values increase the risk of volatilization, even with the larger beam size. Standards used for $\mathrm{Si}, \mathrm{Al}, \mathrm{Fe}, \mathrm{Mg}, \mathrm{Ca}, \mathrm{Na}$, and $\mathrm{Ti}$ are fresh basaltic glass dredged from the Juan de Fuca Ridge (USNM111240/52; VG-2) and Smithsonian standard microcline and apatite for $\mathrm{K}$ and $\mathrm{P}$, respectively. In addition to matrix correction effects and drift corrections, the results are normalized every ten analyses by running the basaltic glass standard. The internal standard is mounted in the center of a 1-inch plastic disk containing ten unknowns around its rim, and eliminates variations caused, for example, by differing thickness of carbon coats.

The homogeneity of the glasses from a single unit and the precision of the glass analyses can be estimated by looking at the variation in results from Unit $\mathrm{A}_{3}$, Hole 395A, a 207-meter-thick pillow lava sequence. Below, the range for each element in the eight glasses analyzed from this unit, the thickest discovered on Leg 45 , is compared with the precision obtained by repeated analyses of standard basaltic glass VG-2 reported previously (Melson et al., 1976):

\begin{tabular}{|c|c|c|c|c|c|c|}
\hline & $\mathrm{SiO}_{2}$ & $\mathrm{Al}_{2} \mathrm{O}_{3}$ & $\mathrm{FeO}^{*}$ & $\underline{\mathrm{MgO}}$ & $\underline{\mathrm{CaO}}$ & $\mathrm{Na}_{2} \mathrm{O}$ \\
\hline \multirow{3}{*}{$\begin{array}{l}\mathrm{A}_{3} \\
\mathrm{VG} 2\end{array}$} & 0.99 & 0.30 & 0.49 & 0.74 & 0.20 & 0.14 \\
\hline & 0.70 & 0.47 & 0.34 & 0.22 & 0.30 & \\
\hline & $\mathrm{K}_{2} \mathrm{O}$ & $\mathrm{TiO}_{2}$ & $\mathrm{P}_{2} \mathrm{O}_{5}$ & & & \\
\hline & 0.0 & 0.07 & 0.05 & & & \\
\hline & 0.04 & 0.12 & 0.04 & & & \\
\hline
\end{tabular}

The range for $\mathrm{MgO}$ is considerably higher than our analytical precision. The variation in the remaining elements is close to or less than (for $\mathrm{CaO}$ and $\mathrm{TiO}_{2}$ ) the precision for VG-2.

The range for each group with five or more analyses is a useful figure in distinguishing significant chemical 
TABLE 1

Basaltic Glass Analyses, Hole 395

\begin{tabular}{|c|c|c|c|c|c|c|c|c|c|c|c|c|c|c|c|}
\hline 1 & 2 & $\mathrm{SiO}_{2}$ & $\mathrm{Al}_{2} \mathrm{O}_{3}$ & $\mathrm{FeO}^{*}$ & $\mathrm{MgO}$ & $\mathrm{CaO}$ & $\mathrm{Na}_{2} \mathrm{O}$ & $\mathrm{K}_{2} \mathrm{O}$ & $\mathrm{TiO}_{2}$ & $\mathrm{P}_{2} \mathrm{O}_{5}$ & Sum & Core & Section & $\begin{array}{l}\text { Interval } \\
(\mathrm{cm})\end{array}$ & VG. no. \\
\hline $\mathrm{A}_{2}$ & $\mathrm{G}_{1}$ & 50.16 & 15.45 & 10.92 & 7.53 & 10.54 & 3.04 & 0.11 & 1.66 & 0.15 & 99.56 & 11 & 1 & 123 & N50 \\
\hline $\mathrm{A}_{2}$ & $\mathrm{G}_{1}$ & 50.37 & 15.60 & 10.90 & 7.03 & 10.84 & 3.03 & 0.12 & 1.70 & 0.17 & 99.76 & 11 & 1 & 135 & N51 \\
\hline $\mathrm{A}_{2}$ & $\mathrm{G}_{1}$ & 50.50 & 15.76 & 10.89 & 7.00 & 10.60 & 3.14 & 0.10 & 1.67 & 0.15 & 99.81 & 14 & 1 & 19 & N52 \\
\hline$A_{2}$ & $\mathrm{G}_{1}$ & 50.45 & 15.30 & 10.80 & 7.25 & 10.62 & 2.97 & 0.11 & 1.67 & 0.16 & 99.33 & 14 & 1 & 119 & N53 \\
\hline $\mathrm{A}_{2}$ & $\mathrm{G}_{1}$ & 51.31 & 15.65 & 11.01 & 7.07 & 10.84 & 2.84 & 0.13 & 1.63 & 0.16 & 100.64 & 16 & 2 & 102 & N93 \\
\hline $\mathrm{A}_{2}$ & $\mathrm{G}_{1}$ & 50.13 & 15.67 & 11.09 & 7.39 & 10.86 & 2.96 & 0.11 & 1.64 & 0.17 & 100.02 & 16 & 2 & 103 & N54 \\
\hline $\mathrm{P}_{1}$ & $\mathrm{G}_{2}$ & 51.48 & 14.33 & 9.72 & 7.49 & 12.15 & 2.67 & 0.11 & 1.49 & 0.13 & 99.57 & 18 & 2 & 38 & N56 \\
\hline
\end{tabular}

Note: Column 1 is the shipboard XRF type assigned to the interval from which the glass was sampled. $A=$ aphyric units, $P=$ phyric units. Second column is chemical type assigned to glass analyses for this hole only.

TABLE 2

Basaltic Glass Analyses, Hole 395A, Core 5A

\begin{tabular}{|c|c|c|c|c|c|c|c|c|c|c|c|c|c|c|}
\hline 1 & 2 & $\mathrm{SiO}_{2}$ & $\mathrm{Al}_{2} \mathrm{O}_{3}$ & $\mathrm{FeO}^{*}$ & $\mathrm{MgO}$ & $\mathrm{CaO}$ & $\mathrm{Na}_{2} \mathrm{O}$ & $\mathrm{K}_{2} \mathrm{O}$ & $\mathrm{TiO}_{2}$ & $\mathrm{P}_{2} \mathrm{O}_{5}$ & Sum & Core & $\begin{array}{l}\text { Interval } \\
(\mathrm{cm})\end{array}$ & VG. no. \\
\hline - & $\mathrm{G}_{1}$ & 50.24 & 15.10 & 9.87 & 8.06 & 11.02 & 2.72 & 0.11 & 1.65 & 0.14 & 98.91 & $5 \mathrm{~A}$ & $\mathrm{CC}$ & L46 \\
\hline - & $\mathrm{G}_{2}$ & 49.29 & 15.19 & 11.06 & 7.95 & 10.58 & 2.95 & 0.10 & 1.57 & 0.13 & 99.12 & $5 \mathrm{~A}$ & $\mathrm{CC}$ & L40 \\
\hline - & $\mathrm{G}_{2}$ & 49.41 & 15.34 & 10.85 & 8.25 & 10.58 & 2.97 & 0.11 & 1.54 & 0.14 & 99.19 & $5 \mathrm{~A}$ & CC & L41 \\
\hline - & $\mathrm{G}_{2}$ & 49.45 & 15.18 & 10.93 & 8.17 & 10.50 & 2.95 & 0.10 & 1.58 & 0.13 & 98.99 & $5 \mathrm{~A}$ & $\mathrm{CC}$ & L42 \\
\hline - & $\mathrm{G}_{2}$ & 49.97 & 15.33 & 10.97 & 7.86 & 10.54 & 2.95 & 0.11 & 1.60 & 0.14 & 99.47 & $5 \mathrm{~A}$ & $\mathrm{CC}$ & L43 \\
\hline 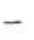 & $\mathrm{G}_{2}$ & 49.18 & 15.38 & 11.09 & 8.01 & 10.55 & 2.93 & 0.12 & 1.58 & 0.12 & 98.96 & $5 \mathrm{~A}$ & $\mathrm{CC}$ & L44 \\
\hline - & $\mathrm{G}_{2}$ & 50.21 & 15.37 & 11.01 & 7.97 & 10.56 & 2.95 & 0.10 & 1.66 & 0.15 & 99.99 & $5 \mathrm{~A}$ & $\mathrm{CC}$ & L45 \\
\hline - & $\mathrm{G}_{2}$ & 49.81 & 15.54 & 10.97 & 7.95 & 10.45 & 2.99 & 0.09 & 1.60 & 0.14 & 99.54 & $5 \mathrm{~A}$ & $\mathrm{CC}$ & L47 \\
\hline - & $\mathrm{G}_{2}$ & 49.87 & 15.40 & 10.95 & 7.99 & 10.58 & 2.93 & 0.11 & 1.56 & 0.13 & 99.52 & $5 \mathrm{~A}$ & $\mathrm{CC}$ & L48 \\
\hline - & $\mathrm{G}_{2}$ & 49.58 & 15.35 & 11.07 & 7.96 & 10.46 & 2.98 & 0.12 & 1.64 & 0.12 & 99.28 & $5 \mathrm{~A}$ & $\mathrm{CC}$ & L49 \\
\hline
\end{tabular}

Note: Column " 1 " is shipboard XRF equivalent (none) and Column " 2 " is glass chemical type.

breaks from apparent breaks caused by analytical precision $(N=$ number of analyses $)$ :

\begin{tabular}{|c|c|c|c|c|c|c|}
\hline Group & $\mathrm{N}$ & $\mathrm{SiO}_{2}$ & $\mathrm{Al}_{2} \mathrm{O}_{3}$ & $\mathrm{FeO} *$ & $\mathrm{MgO}$ & $\mathrm{CaO}$ \\
\hline $\mathrm{G}_{1}(395)$ & 6 & 1.18 & 0.31 & 0.29 & 0.53 & 0.32 \\
\hline $\mathrm{G}_{5}(395 \mathrm{~A})$ & 5 & 0.55 & 0.25 & 0.36 & 0.34 & 0.21 \\
\hline $\mathrm{G}_{7}(395 \mathrm{~A})$ & 8 & 0.99 & 0.47 & 0.49 & 0.74 & 0.20 \\
\hline $\mathrm{G}_{8}(395 \mathrm{~A})$ & 9 & 1.03 & 0.35 & 0.24 & 0.56 & 0.32 \\
\hline $\mathrm{G}_{1}$ (396) & 5 & 0.41 & 0.34 & 0.50 & 0.43 & 0.23 \\
\hline $\mathrm{G}_{2}(396)$ & 11 & 0.85 & 0.47 & 0.52 & 0.42 & 0.22 \\
\hline $\mathrm{G}_{3}(396)$ & 6 & 0.71 & 0.31 & 0.23 & 0.28 & 0.16 \\
\hline \multirow[t]{2}{*}{ Average } & & 0.82 & 0.36 & 0.38 & 0.47 & 0.24 \\
\hline & $\underline{\mathrm{Na}_{2} \mathrm{O}}$ & $\underline{\mathrm{K}_{2} \mathrm{O}}$ & $\underline{\mathrm{TiO}_{2}}$ & $\underline{\mathrm{P}_{2} \mathrm{O} 5}$ & & \\
\hline $\mathrm{G}_{1}(395)$ & 0.30 & 0.03 & 0.07 & 0.02 & & \\
\hline $\mathrm{G}_{5}(395 \mathrm{~A})$ & 0.15 & 0.02 & 0.07 & 0.03 & & \\
\hline $\mathrm{G}_{7}(395 \mathrm{~A})$ & 0.14 & 0.04 & 0.07 & 0.05 & & \\
\hline $\mathrm{G}_{8}(395 \mathrm{~A})$ & 0.13 & 0.01 & 0.15 & 0.04 & & \\
\hline $\mathrm{G}_{1}(396)$ & 0.12 & 0.02 & 0.09 & 0.05 & & \\
\hline $\mathrm{G}_{2}(396)$ & 0.13 & 0.02 & 0.10 & 0.04 & & \\
\hline $\mathrm{G}_{3}(396)$ & 0.11 & 0.02 & 0.09 & 0.04 & & \\
\hline Average & 0.15 & 0.02 & 0.09 & 0.04 & & \\
\hline
\end{tabular}

A comparison between the XRF and EMX analyses reveals good agreement after appropriate corrections are made. Below is a comparison, where $N$ is number of samples analyzed, type is shipboard chemical group $\mathrm{XRF}$ analysis (A or $\mathrm{P}$ ) and equivalent glass group, $\mathrm{G}$ :

\begin{tabular}{|c|c|c|c|c|c|c|}
\hline Type & $\mathrm{N}$ & $\mathrm{SiO}_{2}$ & $\mathrm{Al}_{2} \mathrm{O}_{3}$ & $\mathrm{FeO} *$ & $\mathrm{MgO}$ & $\mathrm{CaO}$ \\
\hline$A_{2}$ & 12 & 49.31 & 14.97 & 10.96 & 8.56 & 10.57 \\
\hline $\mathrm{G}_{1}(395)$ & & & & & & \\
\hline $\mathrm{G}_{3}(395 \mathrm{~A})$ & 7 & 50.51 & 15.61 & 10.96 & 7.18 & 10.75 \\
\hline $\mathrm{A}_{3}$ & 16 & 49.73 & 15.14 & 10.08 & 7.61 & 11.29 \\
\hline $\mathrm{G}_{7}(395 \mathrm{~A})$ & 8 & 50.92 & 15.49 & 9.86 & 7.25 & 11.20 \\
\hline $\mathrm{A}_{4}$ & 8 & 49.14 & 15.09 & 10.19 & 8.30 & 11.03 \\
\hline \multirow[t]{2}{*}{$\mathrm{Gg}_{9}(395 \mathrm{~A})$} & 9 & 50.05 & 15.52 & 10.22 & 8.05 & 11.01 \\
\hline & $\mathrm{Na}_{2} \mathrm{O}$ & $\mathrm{K}_{2} \mathrm{O}$ & $\underline{\mathrm{TiO}_{2}}$ & $\underline{\mathrm{P}_{2} \mathrm{O}_{5}}$ & & \\
\hline $\mathrm{A}_{2}$ & 2.85 & 0.12 & 1.63 & 0.16 & & \\
\hline \multicolumn{7}{|l|}{$\mathrm{G}_{1}$ (395) } \\
\hline $\mathrm{G}_{3}(395 \mathrm{~A})$ & 2.99 & 0.10 & 1.66 & 0.17 & & \\
\hline $\mathrm{A}_{3}$ & 2.68 & 0.21 & 1.72 & 0.17 & & \\
\hline $\mathrm{G}_{7}(395 \mathrm{~A})$ & 2.90 & 0.13 & 1.72 & 0.17 & & \\
\hline $\mathrm{A}_{4}$ & 2.66 & 0.22 & 1.61 & 0.15 & & \\
\hline G9 (395A) & 2.84 & 0.11 & 1.61 & 0.14 & & \\
\hline
\end{tabular}

For direct comparison with XRF analyses, the EMX analyses can be corrected for at least three effects: (1) dilution effect caused by presence of $\mathrm{H}_{2} \mathrm{O}$ and other volatiles, (2) dilution effect of addition of oxygen to oxidize ferrous iron to ferric iron during fusion for XRF analyses, and (3) presence of olivine microphenocrysts. Below is a corrected analysis of the average glass analyses for the $\mathrm{A}_{2}$ equivalent group. Corrections are made assuming the presence of 0.4 per cent $\mathrm{H}_{2} \mathrm{O}$ in 
TABLE 3

Basaltic Glass Analyses, Hole 395A

\begin{tabular}{|c|c|c|c|c|c|c|c|c|c|c|c|c|c|c|c|}
\hline 1 & 2 & $\mathrm{SiO}_{2}$ & $\mathrm{Al}_{2} \mathrm{O}_{3}$ & $\mathrm{FeO}^{*}$ & $\mathrm{MgO}$ & $\mathrm{CaO}$ & $\mathrm{Na}_{2} \mathrm{O}$ & $\mathrm{K}_{2} \mathrm{O}$ & $\mathrm{TiO}_{2}$ & $\mathrm{P}_{2} \mathrm{O}_{5}$ & Sum & Core & Section & $\begin{array}{c}\text { Interval } \\
(\mathrm{cm})\end{array}$ & VG. no. \\
\hline- & $\mathrm{G}_{1}$ & 50.24 & 15.10 & 9.87 & 8.06 & 11.02 & 2.72 & 0.11 & 1.65 & 0.14 & 98.91 & $5 \mathrm{~A}$ & $\mathrm{CC}$ & & L46 \\
\hline- & $\mathrm{G}_{2}$ & 49.64 & 15.33 & 10.98 & 8.01 & 10.53 & 2.95 & 0.11 & 1.52 & 0.13 & 99.20 & $5 \mathrm{~A}$ & $\mathrm{CC}$ & & * \\
\hline $\mathrm{A}_{2}$ & $\mathrm{G}_{3}$ & 50.58 & 15.85 & 11.07 & 6.97 & 10.89 & 2.96 & 0.10 & 1.68 & 0.16 & 100.27 & 11 & 1 & 74 & N57 \\
\hline $\begin{array}{l}\mathrm{P}_{2} \\
\mathrm{P}_{3} \\
\mathrm{P}_{3} \\
\end{array}$ & $\begin{array}{l}\mathrm{G}_{4} \\
\mathrm{G}_{4} \\
\mathrm{G}_{4}\end{array}$ & $\begin{array}{l}51.73 \\
51.18 \\
51.94 \\
\end{array}$ & $\begin{array}{l}14.92 \\
14.81 \\
15.11 \\
\end{array}$ & $\begin{array}{r}9.74 \\
9.50 \\
10.11 \\
\end{array}$ & $\begin{array}{l}7.57 \\
7.40 \\
7.53 \\
\end{array}$ & $\begin{array}{l}12.20 \\
12.30 \\
12.40 \\
\end{array}$ & $\begin{array}{l}2.46 \\
2.48 \\
2.44 \\
\end{array}$ & $\begin{array}{l}0.09 \\
0.12 \\
0.08 \\
\end{array}$ & $\begin{array}{l}1.41 \\
1.39 \\
1.40 \\
\end{array}$ & $\begin{array}{l}0.18 \\
0.13 \\
0.15 \\
\end{array}$ & $\begin{array}{r}100.30 \\
99.30 \\
101.16 \\
\end{array}$ & $\begin{array}{l}16 \\
17 \\
20 \\
\end{array}$ & $\begin{array}{l}1 \\
1 \\
1 \\
\end{array}$ & $\begin{array}{l}87 \\
84 \\
42 \\
\end{array}$ & $\begin{array}{l}\text { N94 } \\
\text { N95 } \\
\text { N96 } \\
\end{array}$ \\
\hline $\begin{array}{l}\mathrm{P}_{4} \\
\mathrm{P}_{4} \\
\mathrm{P}_{4} \\
\mathrm{P}_{4} \\
\mathrm{P}_{4}\end{array}$ & $\begin{array}{l}\mathrm{G}_{5} \\
\mathrm{G}_{5} \\
\mathrm{G}_{5} \\
\mathrm{G}_{5} \\
\mathrm{G}_{5}\end{array}$ & $\begin{array}{l}51.44 \\
51.36 \\
51.75 \\
51.69 \\
51.22 \\
\end{array}$ & $\begin{array}{l}14.86 \\
14.74 \\
14.88 \\
14.63 \\
14.73\end{array}$ & $\begin{array}{l}9.80 \\
9.77 \\
9.47 \\
9.57 \\
9.44 \\
\end{array}$ & $\begin{array}{l}7.29 \\
7.30 \\
6.86 \\
7.03 \\
6.96 \\
\end{array}$ & $\begin{array}{l}11.84 \\
11.77 \\
11.90 \\
11.93 \\
11.98 \\
\end{array}$ & $\begin{array}{l}2.71 \\
2.76 \\
2.86 \\
2.79 \\
2.86\end{array}$ & $\begin{array}{l}0.10 \\
0.12 \\
0.10 \\
0.10 \\
0.11\end{array}$ & $\begin{array}{l}1.48 \\
1.55 \\
1.51 \\
1.52 \\
1.53\end{array}$ & $\begin{array}{l}0.14 \\
0.13 \\
0.16 \\
0.14 \\
0.14 \\
\end{array}$ & $\begin{array}{l}99.66 \\
99.50 \\
99.50 \\
99.40 \\
98.97\end{array}$ & $\begin{array}{l}23 \\
26 \\
26 \\
26 \\
27 \\
\end{array}$ & $\begin{array}{l}1 \\
2 \\
2 \\
2 \\
2\end{array}$ & $\begin{array}{r}84 \\
75 \\
89 \\
124 \\
69 \\
\end{array}$ & $\begin{array}{l}\text { N97 } \\
\text { N99 } \\
\text { P10 } \\
\text { P11 } \\
\text { P12 }\end{array}$ \\
\hline$P_{5}$ & $\mathrm{G}_{6}$ & 50.63 & 15.22 & 9.63 & 6.91 & 11.60 & 3.08 & 0.12 & 1.62 & 0.14 & 98.95 & 30 & 1 & 33 & P13 \\
\hline $\begin{array}{l}\mathrm{A}_{3} \\
\mathrm{~A}_{3} \\
\mathrm{~A}_{3} \\
\mathrm{~A}_{3} \\
\mathrm{~A}_{3} \\
\mathrm{~A}_{3} \\
\mathrm{~A}_{3} \\
\mathrm{~A}_{3} \\
\end{array}$ & $\begin{array}{l}\mathrm{G}_{7} \\
\mathrm{G}_{7} \\
\mathrm{G}_{7} \\
\mathrm{G}_{7} \\
\mathrm{G}_{7} \\
\mathrm{G}_{7} \\
\mathrm{G}_{7} \\
\mathrm{G}_{7}\end{array}$ & $\begin{array}{l}51.25 \\
50.97 \\
51.16 \\
51.38 \\
51.23 \\
50.52 \\
50.46 \\
50.39 \\
\end{array}$ & \begin{tabular}{l|}
15.59 \\
15.28 \\
15.37 \\
15.42 \\
15.54 \\
15.75 \\
15.58 \\
15.40 \\
\end{tabular} & $\begin{array}{r}9.61 \\
9.77 \\
10.10 \\
9.77 \\
9.87 \\
10.07 \\
9.88 \\
9.83 \\
\end{array}$ & $\begin{array}{l}7.06 \\
7.54 \\
7.68 \\
6.82 \\
7.50 \\
6.94 \\
7.02 \\
7.46 \\
\end{array}$ & $\begin{array}{l}11.15 \\
11.18 \\
11.35 \\
11.25 \\
11.16 \\
11.19 \\
11.29 \\
10.99 \\
\end{array}$ & $\begin{array}{l}2.97 \\
2.88 \\
2.83 \\
2.94 \\
2.89 \\
2.93 \\
2.89 \\
2.90 \\
\end{array}$ & $\begin{array}{l}0.14 \\
0.15 \\
0.13 \\
0.16 \\
0.12 \\
0.12 \\
0.11 \\
0.11 \\
\end{array}$ & $\begin{array}{l}1.70 \\
1.72 \\
1.70 \\
1.80 \\
1.68 \\
1.75 \\
1.74 \\
1.73 \\
\end{array}$ & $\begin{array}{l}0.16 \\
0.14 \\
0.17 \\
0.19 \\
0.21 \\
0.16 \\
0.17 \\
0.15 \\
\end{array}$ & $\begin{array}{r}99.63 \\
99.63 \\
100.49 \\
99.73 \\
100.20 \\
99.43 \\
99.14 \\
98.96 \\
\end{array}$ & $\begin{array}{l}35 \\
35 \\
41 \\
51 \\
51 \\
52 \\
53 \\
53 \\
\end{array}$ & $\begin{array}{l}1 \\
1 \\
1 \\
1 \\
2 \\
2 \\
1 \\
1 \\
2\end{array}$ & $\begin{array}{r}60 \\
142 \\
73 \\
50 \\
112 \\
120 \\
10 \\
149 \\
\end{array}$ & $\begin{array}{l}\text { P14 } \\
\text { P15 } \\
\text { P16 } \\
\text { N58 } \\
\text { N59 } \\
\text { N60 } \\
\text { N61 } \\
\text { N62 } \\
\end{array}$ \\
\hline $\begin{array}{l}\mathrm{A}_{4} \\
\mathrm{~A}_{4} \\
\mathrm{~A}_{4} \\
\mathrm{~A}_{4} \\
\mathrm{~A}_{4} \\
\mathrm{~A}_{4} \\
\mathrm{~A}_{4} \\
\mathrm{~A}_{4} \\
\mathrm{~A}_{4}\end{array}$ & $\begin{array}{l}\mathrm{G}_{8} \\
\mathrm{G}_{8} \\
\mathrm{G}_{8} \\
\mathrm{G}_{8} \\
\mathrm{G}_{8} \\
\mathrm{G}_{8} \\
\mathrm{G}_{8} \\
\mathrm{G}_{8} \\
\mathrm{G}_{8}\end{array}$ & $\begin{array}{l}50.22 \\
50.45 \\
49.58 \\
49.77 \\
50.20 \\
50.14 \\
50.28 \\
49.66 \\
50.19 \\
\end{array}$ & $\begin{array}{l}15.64 \\
15.69 \\
15.47 \\
15.57 \\
15.35 \\
15.65 \\
15.52 \\
15.34 \\
15.51 \\
\end{array}$ & $\begin{array}{l}10.11 \\
10.25 \\
10.09 \\
10.27 \\
10.33 \\
10.33 \\
10.28 \\
10.15 \\
10.19 \\
\end{array}$ & $\begin{array}{l}8.07 \\
8.19 \\
8.08 \\
8.31 \\
8.06 \\
7.69 \\
8.25 \\
7.91 \\
8.07 \\
\end{array}$ & $\begin{array}{l}11.05 \\
11.08 \\
10.82 \\
11.01 \\
11.04 \\
11.09 \\
11.14 \\
11.00 \\
10.87 \\
\end{array}$ & $\begin{array}{l}2.84 \\
2.91 \\
2.84 \\
2.87 \\
2.87 \\
2.79 \\
2.78 \\
2.85 \\
2.89\end{array}$ & $\begin{array}{l}0.11 \\
0.12 \\
0.11 \\
0.11 \\
0.12 \\
0.12 \\
0.12 \\
0.11 \\
0.11 \\
\end{array}$ & $\begin{array}{l}1.62 \\
1.64 \\
1.55 \\
1.60 \\
1.60 \\
1.60 \\
1.58 \\
1.62 \\
1.70\end{array}$ & $\begin{array}{l}0.14 \\
0.15 \\
0.15 \\
0.15 \\
0.14 \\
0.15 \\
0.12 \\
0.14 \\
0.16 \\
\end{array}$ & $\begin{array}{r}99.80 \\
100.48 \\
98.69 \\
99.65 \\
99.71 \\
99.56 \\
100.07 \\
98.78 \\
99.70 \\
\end{array}$ & $\begin{array}{l}57 \\
58 \\
58 \\
58 \\
59 \\
60 \\
60 \\
64 \\
66 \\
\end{array}$ & $\begin{array}{l}1 \\
2 \\
2 \\
2 \\
1 \\
1 \\
1 \\
3 \\
2 \\
1 \\
\end{array}$ & $\begin{array}{r}136 \\
83 \\
97 \\
100 \\
103 \\
115 \\
125 \\
129 \\
106 \\
\end{array}$ & $\begin{array}{l}\text { N63 } \\
\text { P17 } \\
\text { N64 } \\
\text { N65 } \\
\text { P18 } \\
\text { N67 } \\
\text { N68 } \\
\text { N69 } \\
\text { P19 } \\
\end{array}$ \\
\hline $\mathrm{A}_{5}$ & $\mathrm{G}_{9}$ & 50.39 & 15.82 & 10.22 & 7.38 & 11.24 & 2.92 & 0.12 & 1.68 & 0.14 & 99.91 & 67 & 1 & 145 & P20 \\
\hline $\mathrm{A}_{5}$ & $\mathrm{G}_{10}$ & 50.32 & 15.54 & 10.42 & 7.96 & 10.81 & 2.83 & 0.10 & 1.62 & 0.19 & 99.78 & 67 & 2 & 125 & N70 \\
\hline
\end{tabular}

Note: Column 1 is shipboard XRF chemical type assigned to the interval from which the glass was sampled. Column 2 is chemical type of glass assigned in this study. Solid lines are discontinuities in glass chemical types.

TABLE 4

Basaltic Glass Analyses, Hole 396

\begin{tabular}{|c|c|c|c|c|c|c|c|c|c|c|c|c|c|c|}
\hline 1 & $\mathrm{SiO}_{2}$ & $\mathrm{Al}_{2} \mathrm{O}_{3}$ & $\mathrm{FeO}^{*}$ & $\mathrm{MgO}$ & $\mathrm{CaO}$ & $\mathrm{Na}_{2} \mathrm{O}$ & $\mathrm{K}_{2} \mathrm{O}$ & $\mathrm{TiO}_{2}$ & $\mathrm{P}_{2} \mathrm{O}_{5}$ & Sum & Core & Section & $\begin{array}{c}\text { Interval } \\
(\mathrm{cm})\end{array}$ & VG. no. \\
\hline $\mathrm{G}_{1}$ & 51.68 & 15.57 & 9.78 & 7.42 & 11.41 & 2.90 & 0.12 & 1.69 & 0.19 & 100.76 & 14 & 6 & 43 & N79 \\
\hline $\mathrm{G}_{1}$ & 50.50 & 15.70 & 9.28 & 6.99 & 11.18 & 2.99 & 0.13 & 1.65 & 0.15 & 98.57 & 14 & 6 & 66 & N80 \\
\hline $\mathrm{G}_{1}$ & 50.56 & 15.36 & 9.49 & 7.31 & 11.32 & 2.87 & 0.12 & 1.60 & 0.14 & 98.77 & 14 & 6 & 66 & P21 \\
\hline $\mathrm{G}_{1}$ & 50.79 & 15.62 & 9.69 & 7.22 & 11.36 & 2.94 & 0.14 & 1.65 & 0.18 & 99.59 & 14 & 6 & 70 & N78 \\
\hline $\mathrm{G}_{1}$ & 50.91 & 15.61 & 9.42 & 7.14 & 11.27 & 2.95 & 0.12 & 1.67 & 0.15 & 99.24 & 14 & 6 & 110 & N81 \\
\hline $\mathrm{G}_{2}$ & 50.59 & 16.05 & 8.79 & 7.80 & 11.67 & 2.87 & 0.12 & 1.43 & 0.14 & 99.46 & 15 & 1 & 62 & N83 \\
\hline $\mathrm{G}_{2}$ & 50.72 & 16.22 & 8.87 & 8.09 & 11.49 & 2.74 & 0.11 & 1.37 & 0.14 & 99.75 & 15 & 4 & 93 & N75 \\
\hline $\mathrm{G}_{2}$ & 50.97 & 16.27 & 8.86 & 8.04 & 11.68 & 2.81 & 0.11 & 1.33 & 0.14 & 100.21 & 16 & 1 & 42 & N74 \\
\hline $\mathrm{G}_{2}$ & 50.53 & 16.44 & 8.27 & 8.09 & 11.70 & 2.85 & 0.11 & 1.39 & 0.13 & 99.51 & 16 & 1 & 80 & N84 \\
\hline $\mathrm{G}_{2}^{2}$ & 50.12 & 15.94 & 8.79 & 8.13 & 11.61 & 2.75 & 0.11 & 1.38 & 0.12 & 98.95 & 16 & 2 & 138 & P22 \\
\hline $\mathrm{G}_{2}$ & 50.54 & 16.38 & 8.81 & 7.90 & 11.50 & 2.79 & 0.10 & 1.33 & 0.13 & 99.48 & 16 & 4 & 58 & N71 \\
\hline $\mathrm{G}_{2}$ & 50.88 & 16.40 & 8.79 & 7.89 & 11.71 & 2.82 & 0.11 & 1.41 & 0.13 & 100.14 & 18 & $\mathrm{cc}$ & 50 & N85 \\
\hline $\mathrm{G}_{2}$ & 50.43 & 16.17 & 8.81 & 7.86 & 11.66 & 2.78 & 0.11 & 1.37 & 0.12 & 99.31 & 19 & 2 & 12 & N86 \\
\hline $\mathrm{G}_{2}$ & 50.47 & 16.34 & 8.65 & 7.71 & 11.60 & 2.86 & 0.10 & 1.34 & 0.16 & 99.23 & 21 & 1 & 42 & $\mathrm{~N} 72$ \\
\hline $\mathrm{G}_{2}$ & 50.49 & 16.25 & 8.72 & 7.91 & 11.50 & 2.84 & 0.11 & 1.33 & 0.16 & 99.31 & 22 & 1 & 125 & N73 \\
\hline $\mathrm{G}_{2}$ & 50.33 & 16.43 & 8.72 & 7.93 & 11.71 & 2.81 & 0.11 & 1.36 & 0.14 & 99.54 & 22 & 2 & 13 & N87 \\
\hline $\mathrm{G}_{3}$ & 50.96 & 15.10 & 9.84 & 7.37 & 11.72 & 2.76 & 0.10 & 1.55 & 0.14 & 99.54 & 22 & 4 & 100 & N88 \\
\hline $\mathrm{G}_{3}$ & 50.77 & 15.32 & 9.91 & 7.35 & 11.56 & 2.71 & 0.10 & 1.55 & 0.14 & 99.41 & 23 & 1 & 75 & N89 \\
\hline $\mathrm{G}_{3}$ & 51.00 & 15.30 & 9.78 & 7.32 & 11.61 & 2.72 & 0.10 & 1.53 & 0.17 & 99.53 & 24 & 1 & 78 & N76 \\
\hline $\mathrm{G}_{3}$ & 51.24 & 15.13 & 10.01 & 7.46 & 11.68 & 2.65 & 0.11 & 1.46 & 0.14 & 99.88 & 24 & 2 & 112 & N90 \\
\hline $\mathrm{G}_{3}$ & 51.48 & 15.11 & 9.88 & 7.55 & 11.66 & 2.69 & 0.10 & 1.53 & 0.17 & 100.17 & 24 & 3 & 74 & N91 \\
\hline $\mathrm{G}_{3}$ & 51.45 & 15.01 & 9.99 & $7: 60$ & 11.65 & 2.69 & 0.12 & 1.55 & 0.18 & 100.24 & 25 & 1 & 40 & N92 \\
\hline
\end{tabular}

Note: Column 1 is basaltic glass chemical type. Shipboard XRF analyses were considered as members of one group. Solid lines where discontinuities occur between chemical groups. 
TABLE 5

Summary of Glass Chemical Types from Holes 395, 395 A, and 396, in Stratigraphic Sequence (top to bottom)

\begin{tabular}{|c|c|c|c|c|c|c|c|c|c|c|c|c|c|c|}
\hline Site & $\mathrm{N}$ & $\mathrm{E}$ & $\mathrm{SiO}_{2}$ & $\mathrm{Al}_{2} \mathrm{O}_{3}$ & $\mathrm{FeO}^{*}$ & $\mathrm{MgO}$ & $\mathrm{CaO}$ & $\mathrm{Na}_{2} \mathrm{O}$ & $\mathrm{K}_{2} \mathrm{O}$ & $\mathrm{TiO}_{2}$ & $\mathrm{P}_{2} \mathrm{O}_{5}$ & Sum & Cores & $\mathrm{T}(\mathrm{m})$ \\
\hline $\mathrm{G}_{1}(395)$ & 6 & $\mathrm{~A}_{2}$ & $\begin{array}{r}50.49 \\
1.18\end{array}$ & $\begin{array}{r}15.57 \\
0.31\end{array}$ & $\begin{array}{r}10.94 \\
0.29\end{array}$ & $\begin{array}{l}7.21 \\
0.53\end{array}$ & $\begin{array}{r}10.72 \\
0.32\end{array}$ & $\begin{array}{l}3.00 \\
0.30\end{array}$ & $\begin{array}{l}0.11 \\
0.03\end{array}$ & $\begin{array}{l}1.66 \\
0.07\end{array}$ & $\begin{array}{l}0.16 \\
0.02\end{array}$ & 99.86 & $10-17$ & 66 \\
\hline $\mathrm{G}_{2}(395)$ & 1 & $P_{1}$ & 51.48 & 14.33 & 7.49 & 6.90 & 12.15 & 2.67 & 0.11 & 1.49 & 0.13 & 99.57 & $18-20$ & 25 \\
\hline $\begin{array}{l}\mathrm{G}_{1}(395 \mathrm{~A}) \\
\mathrm{G}_{2}(395 \mathrm{~A}) \\
\mathrm{G}_{3}(395 \mathrm{~A}) \\
\mathrm{G}_{4}(395 \mathrm{~A})\end{array}$ & $\begin{array}{l}1 \\
1 \\
1 \\
3\end{array}$ & $\begin{array}{c}- \\
\overline{\mathrm{A}_{2}} \\
\mathrm{P}_{2}-\mathrm{P}_{3}\end{array}$ & $\begin{array}{r}50.24 \\
49.64 \\
50.58 \\
51.62 \\
0.76\end{array}$ & $\begin{array}{r}15.10 \\
15.33 \\
15.85 \\
14.95 \\
0.30\end{array}$ & $\begin{array}{r}9.87 \\
10.98 \\
11.07 \\
9.78 \\
0.37\end{array}$ & $\begin{array}{l}8.06 \\
8.01 \\
6.97 \\
7.50 \\
0.17\end{array}$ & $\begin{array}{r}11.02 \\
10.53 \\
10.89 \\
12.30 \\
0.20\end{array}$ & $\begin{array}{l}2.72 \\
2.95 \\
2.96 \\
2.46 \\
0.04\end{array}$ & $\begin{array}{l}0.11 \\
0.11 \\
0.10 \\
0.10 \\
0.04\end{array}$ & $\begin{array}{l}1.65 \\
1.52 \\
1.68 \\
1.40 \\
0.02\end{array}$ & $\begin{array}{l}0.14 \\
0.13 \\
0.16 \\
0.15 \\
0.05\end{array}$ & $\begin{array}{r}98.91 \\
99.20 \\
100.27 \\
100.26\end{array}$ & $\begin{array}{c}5 A \\
5 A \\
5-12 \\
13-22\end{array}$ & $\begin{array}{l}<9.5 \\
<9.5 \\
66 \\
83\end{array}$ \\
\hline $\mathrm{G}_{5}(395 \mathrm{~A})$ & 5 & $\mathrm{P}_{4}$ & $\begin{array}{r}0.76 \\
51.49 \\
0.55\end{array}$ & $\begin{array}{r}0.30 \\
14.77 \\
0.25\end{array}$ & $\begin{array}{l}0.37 \\
9.61 \\
0.36\end{array}$ & $\begin{array}{l}0.17 \\
7.09 \\
0.34\end{array}$ & $\begin{array}{r}0.20 \\
11.88 \\
0.21\end{array}$ & $\begin{array}{l}0.04 \\
2.80 \\
0.15\end{array}$ & $\begin{array}{l}0.04 \\
0.11 \\
0.02\end{array}$ & $\begin{array}{l}0.02 \\
1.52 \\
0.07\end{array}$ & $\begin{array}{l}0.05 \\
0.14 \\
0.03\end{array}$ & 99.41 & $2-27$ & 52 \\
\hline $\mathrm{G}_{6}(395 \mathrm{~A})$ & 1 & $\mathrm{P}_{5}$ & 50.63 & 15.22 & 9.63 & 6.91 & 11.60 & 3.08 & 0.12 & 1.62 & 0.14 & 98.95 & $28-33$ & 56 \\
\hline $\mathrm{G}_{7}(395 \mathrm{~A})$ & 8 & $\mathrm{~A}_{3}$ & $\begin{array}{r}50.92 \\
0.99\end{array}$ & $\begin{array}{r}15.49 \\
0.47\end{array}$ & $\begin{array}{l}9.86 \\
0.49\end{array}$ & $\begin{array}{l}7.25 \\
0.74\end{array}$ & $\begin{array}{r}11.20 \\
0.20\end{array}$ & $\begin{array}{l}2.90 \\
0.14\end{array}$ & $\begin{array}{l}0.13 \\
0.04\end{array}$ & $\begin{array}{l}1.73 \\
0.07\end{array}$ & $\begin{array}{l}0.17 \\
0.05\end{array}$ & 99.65 & $33-56$ & 207 \\
\hline $\mathrm{G}_{8}(395 \mathrm{~A})$ & 9 & $\mathrm{~A}_{4}$ & $\begin{array}{r}50.05 \\
1.03\end{array}$ & $\begin{array}{r}15.52 \\
0.35\end{array}$ & $\begin{array}{r}10.22 \\
0.24\end{array}$ & $\begin{array}{l}8.05 \\
0.56\end{array}$ & $\begin{array}{r}11.01 \\
0.32\end{array}$ & $\begin{array}{l}2.84 \\
0.13\end{array}$ & $\begin{array}{l}0.11 \\
0.01\end{array}$ & $\begin{array}{l}1.61 \\
0.15\end{array}$ & $\begin{array}{l}0.14 \\
0.04\end{array}$ & 99.55 & $57-60$ & 66 \\
\hline $\begin{array}{l}\mathrm{G}_{9}(395 \mathrm{~A}) \\
\mathrm{G}_{10}(395 \mathrm{~A})\end{array}$ & $\begin{array}{l}1 \\
1\end{array}$ & $\begin{array}{l}\mathrm{A}_{5} \\
\mathrm{~A}_{5}\end{array}$ & $\begin{array}{l}50.39 \\
50.32\end{array}$ & $\begin{array}{l}15.82 \\
15.54\end{array}$ & $\begin{array}{l}10.22 \\
10.42\end{array}$ & $\begin{array}{l}7.38 \\
7.96\end{array}$ & $\begin{array}{l}11.24 \\
10.81\end{array}$ & $\begin{array}{l}2.92 \\
2.83\end{array}$ & $\begin{array}{l}0.12 \\
0.10\end{array}$ & $\begin{array}{l}1.68 \\
1.62\end{array}$ & $\begin{array}{l}0.14 \\
0.19\end{array}$ & $\begin{array}{l}99.91 \\
99.78\end{array}$ & $\begin{array}{l}67 \\
67 \\
\end{array}$ & $\begin{array}{c}<9.5 \\
?\end{array}$ \\
\hline $\mathrm{G}_{1}(396)$ & 5 & - & $\begin{array}{r}50.89 \\
0.41\end{array}$ & $\begin{array}{r}15.57 \\
0.34\end{array}$ & $\begin{array}{l}9.53 \\
0.50\end{array}$ & $\begin{array}{l}7.22 \\
0.43\end{array}$ & $\begin{array}{r}11.31 \\
0.23\end{array}$ & $\begin{array}{l}2.93 \\
0.12\end{array}$ & $\begin{array}{l}0.13 \\
0.02\end{array}$ & $\begin{array}{l}1.65 \\
0.09\end{array}$ & $\begin{array}{l}0.16 \\
0.05\end{array}$ & 99.39 & 14 & $<9.5$ \\
\hline $\mathrm{G}_{2}(396)$ & 11 & 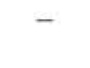 & $\begin{array}{r}50.55 \\
0.85\end{array}$ & $\begin{array}{r}16.26 \\
0.47\end{array}$ & $\begin{array}{l}8.73 \\
0.52\end{array}$ & $\begin{array}{l}7.94 \\
0.42\end{array}$ & $\begin{array}{r}11.62 \\
0.22\end{array}$ & $\begin{array}{l}2.81 \\
0.13\end{array}$ & $\begin{array}{l}0.11 \\
0.02\end{array}$ & $\begin{array}{l}1.36 \\
0.10\end{array}$ & $\begin{array}{l}0.14 \\
0.04\end{array}$ & 99.52 & $15-22$ & 69 \\
\hline $\mathrm{G}_{3}(396)$ & 6 & - & $\begin{array}{r}51.15 \\
0.71\end{array}$ & $\begin{array}{r}15.16 \\
0.31\end{array}$ & $\begin{array}{l}9.90 \\
0.23\end{array}$ & $\begin{array}{l}7.44 \\
0.28\end{array}$ & $\begin{array}{r}11.65 \\
0.16\end{array}$ & $\begin{array}{l}2.70 \\
0.11\end{array}$ & $\begin{array}{l}0.11 \\
0.02\end{array}$ & $\begin{array}{l}1.53 \\
0.09\end{array}$ & $\begin{array}{l}0.16 \\
0.04\end{array}$ & 99.64 & $22-25$ & 16 \\
\hline
\end{tabular}

Note: $N$ is number of samples. $T$ is estimated thickness of unit. $G$ is glass type for the particular site. Where glass type is represented by more than one sample, average and range (second line below average) are given.

the glass, oxidation of 8 per cent $\mathrm{FeO}$, and presence of 3 per cent olivine microphenocrysts with $\mathrm{SiO}_{2}=40.72$ per cent, $\mathrm{FeO}=11.82$ per cent, and $\mathrm{MgO}=45.32$ per cent:

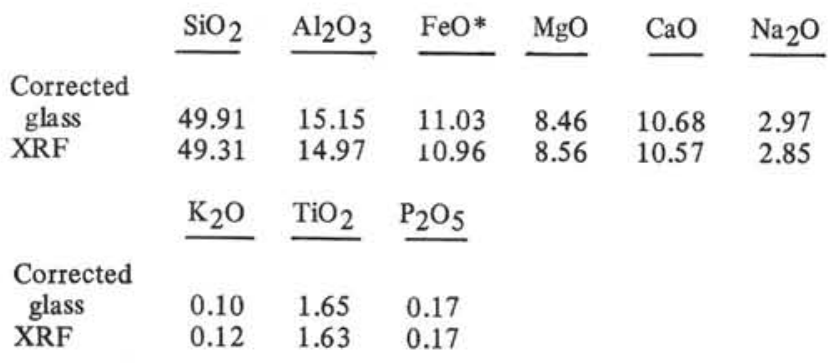

These corrections, made with values consistent with the expected $\mathrm{H}_{2} \mathrm{O}, \mathrm{FeO}$, and olivine content of the fresh glass, bring the XRF and EMX analyses into much closer agreement.

\section{CHEMICAL STRATIGRAPHY: SUMMARY}

In all, the sixty glass analyses define fifteen chemical units (Table 5). These are similar to the shipboard chemical units, but with the following differences:

1) $A_{1}$ (Hole 395) of the shipboard chemical types is not represented by a glass analysis but is a valid unit, possibly a thin intrusive within an $\mathbf{A}_{2}$ sequence in Core 11.

2) Core $5 \mathrm{~A}$, Hole $395 \mathrm{~A}$, contains glasses representative of two units not represented by XRF analyses.

3 ) Three glass analyses from the interval of $P_{2}$ and $P_{3}$ are identical. This suggests that the XRF analyses break into two groups because of systematic differences in the abundance and relative abundance of phenocrysts of plagioclase, olivine, and augite glass.

4) $P_{4}$, glass from the chilled contact of dolerite intrusion near the bottom of Hole 395A, was not sampled and analyzed here.

5) $\mathrm{A}_{5}$ (Hole 395A) is found to be composed of two closely related but distinctly different units.

6) Glasses from Hole 396 are found to fall into three chemical units.

\section{VOLUME RELATIONSHIPS}

A combination of the XRF and EMX chemical stratigraphy gives the following abundances of the units in each hole:

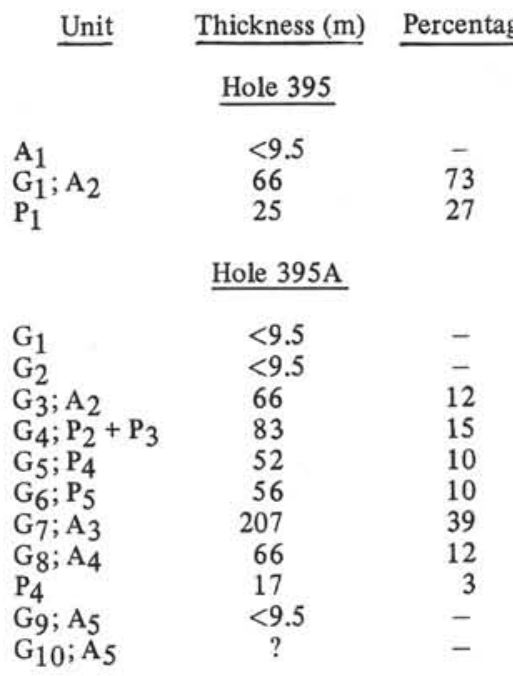




\section{Unit Thickness (m) Percentage}

Hole 396

$\begin{array}{ccc}\mathrm{G}_{1} & 9.5 ? & - \\ \mathrm{G}_{2} & 69 & 81 \\ \mathrm{G}_{3} & >16 & 19\end{array}$

The aphyric basalts are part of a closely related chemical group which, in all, comprise 63 per cent of the cored basement intervals. The phyric rocks comprise about 37 per cent.

\section{REPETITIONS OF CHEMICAL UNITS}

Repetitions of identical chemical magma types do not occur within the units defined on the basis of EMX glass analyses. Some types are extremely close to one another, but are clearly different from one another on the basis of one or more elements. For example, glass units $G_{10}$ (Hole 395A), an aphyric unit, and $G_{1}$ (Hole 396 ), a phyric unit, are extremely close except for lower $\mathrm{FeO}$ in $\mathrm{G}_{1}$ (Hole 396).

\section{ACKNOWLEDGMENTS}

Numerous DSDP personnel of Leg 45 assisted with sampling of the glasses. At the Smithsonian Institution, Tim O'Hearn performed many of the microprobe analyses; Richard Johnson prepared polished sections; and Barbara Keville assisted with manuscript preparation. Tom Wright, U.S. Geological Survey, and Gary Byerly, Smithsonian Institution, assisted with data reduction and provided much useful advice. Eugene Jarosewich continued to improve the accuracy of the composition of VG basalt standard VG-2, to which the accuracies of the analyses here are tied.

\section{REFERENCES}

Byerly, G. R., Melson, W. G., Nelen, J. A., and Jarosewich, E., 1977. Abyssal basaltic glasses as indicators of magma composition, Smithsonian Contrib. to Earth Sci., v. 19, p. 22-30.

Melson, W. G., Vallier, T. L., Wright, T. L., Byerly, G. R., and Nelen, J. A., 1976. Chemical diversity of abyssal volcanic glass erupted along Pacific, Atlantic, and Indian Ocean sea-floor spreading centers, Geophys. Mono. 19, Am. Geophys. Union, p. 351-368. 